\title{
A Dynamic TDMA Protocol Utilizing Channel Sense
}

\author{
ZHOU De-min ${ }^{1, a}$, LIU Yun-jiang ${ }^{2, b}$ and LI Man ${ }^{3, c}$ \\ 1, 2, 3 Information and Navigation College Air Force Engineering University, Xi'an , China \\ azhoudemin1660@163.com, blyj.afeu@126.com, 'Imaf@163.com
}

\begin{abstract}
Keywords: channel sense, TDMA, slot assignment
Abstract. Aimed at the situation that channel source is wasted seriously when traffic is not uniform belong all of the nodes in a TDMA net, a dynamic TDMA protocol (CS-TDMA) utilizing channel sense is was developed. The CS-TDMA protocol based on the TDMA protocol frame, and the node with CS-TDMA protocol counts counted the number of packets from other nodes for estimating their traffic. When the timeslot is remainder, it sends sent an ATS (access to send) message to the busiest node for informing the node to send data until the timeslot was exhaust. Simulation results shown that CS-TDMA protocol had better performance than TDMA protocol on the parameters of end-to-end delay, network throughput and message delivery. Especially, the delay advantage was prominent when the traffic had a big gap between nodes.
\end{abstract}

\section{Introduction}

In a multiple access network, the performances are affected by media access control (MAC) protocol directly, so it is very important to research on the MAC protocol. TDMA protocol is one of the MAC protocols, which is widely used in military field, such as the NATO tactical Link16 and Link22 [1]. In TDMA protocol, time on channel is divided into non-overlapping timeslots, and each member of the network is granted certain slots in which to transmit.

Timeslots of the traditional TDMA protocol are assigned to different nodes in the network initialization stage, and occupied by the nodes all the time. However, when a member doesn't use its entire slot, the unused channel time is wasted. On the contrary, when a member has a large amount of data to be transmitted but its timeslot is not enough. It must wait for the next turn, even if other timeslots are always idle. So, the channel utilization and the delay are bad in a traditional TDMA network, and would be worse when the business is not uniform or real-time changing [2].

In order to improve the channel utilization of TDMA protocol, many papers researched for this problem. The dynamic TDMA protocols improved the channel utilization and the delay performances in a certain extent [3]. The dynamic TDMA protocols could be classified into two categories on the basis of different ways of slots usage: One was a TDMA protocol with fixed slot sizes and flexible allocations, another was a TDMA protocol with changeable slot sizes and unfixed allocation [4][5]. For the first kind, a node could use other nodes' timeslot temporarily after getting authorization when it had much information to be sent, or it could occupy different slots according to its traffic size and priority; For the another, all of the nodes dynamically adjusted their slot size through sending a plan of slot allocation by the control node in the network. Such as, the SNC (system network controller) of Link22 system sent assignment table to every unit to complete the adjustment of timeslots [6]. Both the above methods used additional overhead to achieve the goal of reducing wasted timeslot, but the timeslots might be disordered when the transmission was failed.

Based on TDMA protocol, a kind of dynamic TDMA protocol utilizing channel sense (CS-TDMA) was developed. It used the broadcast characteristic of wireless communication to estimate traffic of other nodes. When timeslot was remainder, the sending node informed the busiest node to transmit data forwardly. Finally, the performance of the CS-TDMA protocol was compared with the TDMA protocol by OPNET simulation. 


\section{The CS-TDMA Protocol}

Design Methodology. In a TDMA network, if a node was in sending state, the other nodes must be in receiving state. Hence, we could assess a node whether make full use of its timeslot through calculating data volume that the node had transmitted. Each node was designed a counter to score the traffic of other nodes, the scoring threshold was set to a fixed value which was under the theoretical maximum transmission bulk. Every node accumulated scores for each of other nodes as a basis for this node to judge which node was the busiest. If the timeslot was surplus, the transmitting node sent an ATS (access to send) message to the busiest node to inform it to occupy the channel until the slot was exhaust. Thus, we achieved the goal that timeslots could be used dynamically according to the traffic of each node, and avoided the case that the timeslots were disordered with the reserved method.

Traffic estimation arithmetic.The exponential increasing method was adopted to score for the node traffic. In the network initialization stage, the SCORE and GAIN parameters were set to 0 and 1 , the Gmax parameter was set to a fixed value which was decided to the capacity of the network. If the traffic from a node was detected exceeding the threshold, score 1 point for this node in first time. If still surpassed the threshold in the next timeframe, add 2 points for this node, and then added 4 points, and so on. On the contrary, reduced the current score value. The specific rule was followed:

step 1: Counted up the traffic named $\mathrm{G}_{\text {slot }}$ from node $\mathrm{N}$ in slot $\mathrm{n}$.

step 2: Compared the $G_{\text {slot }}$ and $G_{\max }$, if $G_{\text {slot }}>G_{\max }$ turned to step 3, otherwise turned to step 4 .

step 3: GAIN $=2 \times$ GAIN; SCORE $=$ SCORE + GAIN; turned to step 5 .

step 4: SCORE = SCORE - GAIN, if SCORE $<=0$, SCORE $=0$, GAIN $=1$.

step 5: Stored the value of SCORE and GAIN for node N, and as the initial value for the next round. Timeslot assignment procedure. At the beginning of a timeslot, the node occupying channel started to send its packets. Then, if all the packets in its storage were sent done but the timeslot was remaining, the node sent an ATS message to the busiest node. Meanwhile, nodes in receiving state got all the messages from channel and scored for their source node. If a node received an ACK message whose destination address was same to local address, this node needed to send its packets immediately until the time slot was used up. The specific slot allocation process was shown as Fig.1.

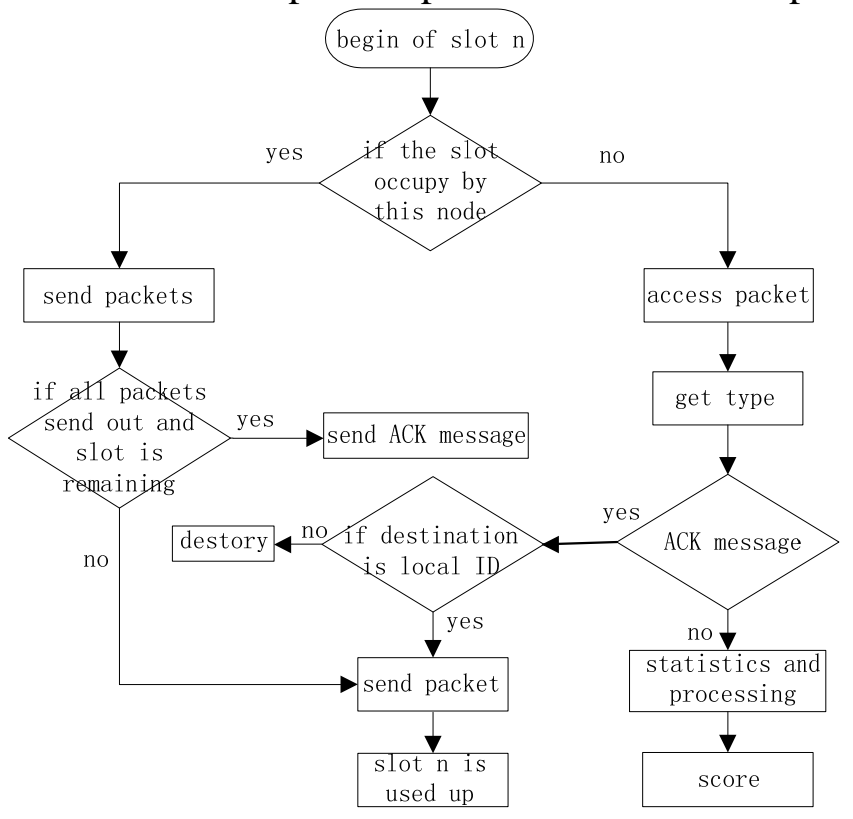

Fig.1 Timeslot assignment procedures of CS-TDMA 


\section{Simulation}

Network model is comprised of node models while node model is made of up process models in the OPNET simulation platform. A certain number of nodes are distributed in a suitable range in network model representing for the topology of real network.

Network model and node model.There are twelve nodes distributing in the area of about 60 kilometers by 80 kilometers. The node model is composed of four parts: source process, CS_TDMA queue, transmitting antenna (rt) and receiving antenna (rr). The source process simulates data generating and traffic statistics. The CS-TDMA queue is responsible for data caching, timeslot allocation and controlling nodes to access channel, which is the major part of the protocol. The RT process is responsible for sending data to wireless channel and the RX process is responsible for receiving data from wireless channel. The network model and node model were shown in Fig.2.

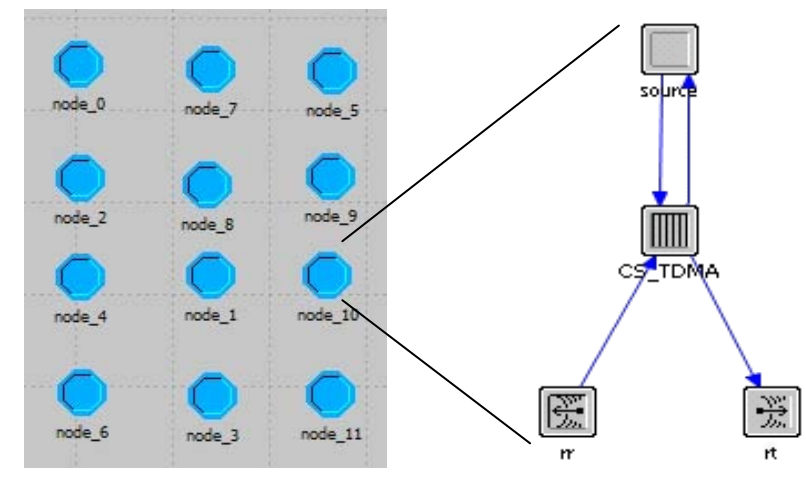

Fig.2 Network model and node model

CS-TDMA process model.According to the design methodology, the CS-TDMA process model was established as shown in Fig.3. Some definitions and explanations were below:

init (initial state): Executed this state for initialing all the parameters firstly when the process starts. Stream_intr (source interrupt state): Get packets which are generated by source process from idle state and inserted them into the data queue.

Slot_intr (slot interrupt state): Set interrupt in this state for dividing time into timeslots and judging which node occupied current timeslot.

Receive (receiving data): Get packets which are were generated by other node from idle state and disposed them according to message types.

Transmit (transmitting data): Send Sent packets which generated by source process from data queue to transmitting antenna.

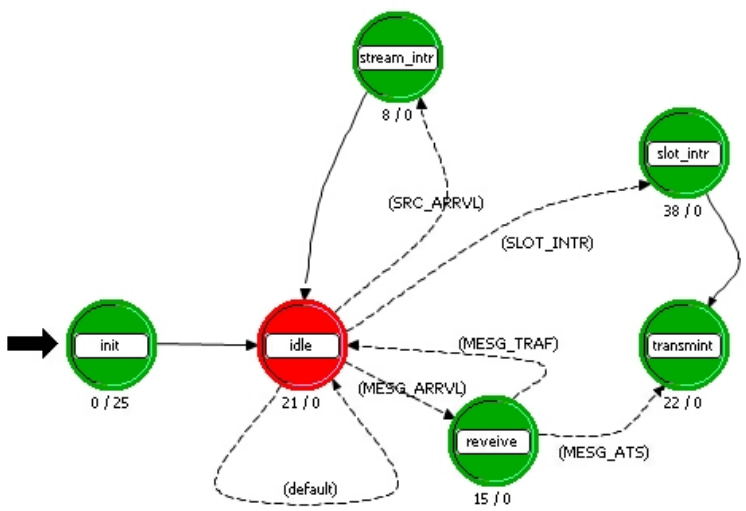

Fig.3 CS-TDMA process model 


\section{Performance Analysis}

The performance of CS-TDMA protocol was analyst by contrasting with the TDMA protocol. In the first scenario, every node had same traffic level, we modified time interval of producing data in source process to simulate different load in real network. In the second scenario, nodes were divided into two parts with different traffic levels, one was designed to be flexible and another was busy and fixed. We simulated the case that nodes in a network had different traffic levels.

QoS Performance in Scenarios1 .Supposed the packet arrival obeyed the Poisson distribution, and set up different packet arrival intervals for $0.1 \mathrm{~s} 、 0.25 \mathrm{~s} 、 0.5 \mathrm{~s} 、 1 \mathrm{~s} 、 2 \mathrm{~s} 、 3 \mathrm{~s} 、 4 \mathrm{~s} 、 5 \mathrm{~s}$. Data transmission rate was $9.6 \mathrm{kbit} / \mathrm{s}$. The business packet size was 200bit and the ATS message size was 32bit.The threshold $G_{\max }$ was 5000bit. The timeslot length was one second. The simulation duration was

Message Delivery Rate

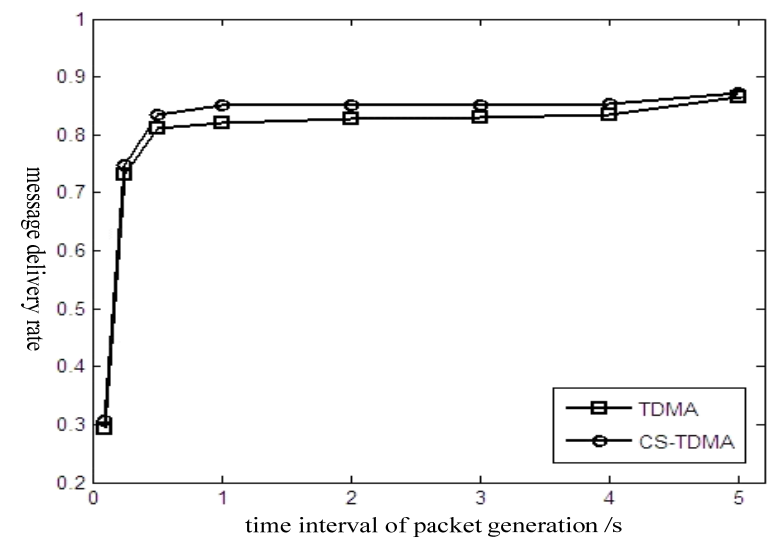

Fig.4 Comparison of message delivery

As shown in Fig.4, when the data generation interval time was $0.25 \mathrm{~s}$, the theoretical value of network load was 9600bit/s that equal to the maximal transmission rate. Due to the channel could not be fully used, network was in congested state. The message delivery rates of two kind protocols were almost same. When the time interval was above $0.5 \mathrm{~s}$, the network was no longer congested and some timeslots were idle in short time. The CS-TDMA protocol could use idle timeslot by sending ATS message to the busiest node dynamically, while the TDMA protocol only could use fixed timeslot that allocated in the initial stage. As a whole, the CS-TDMA protocol had a better performance of deliver rata than TDMA protocol.

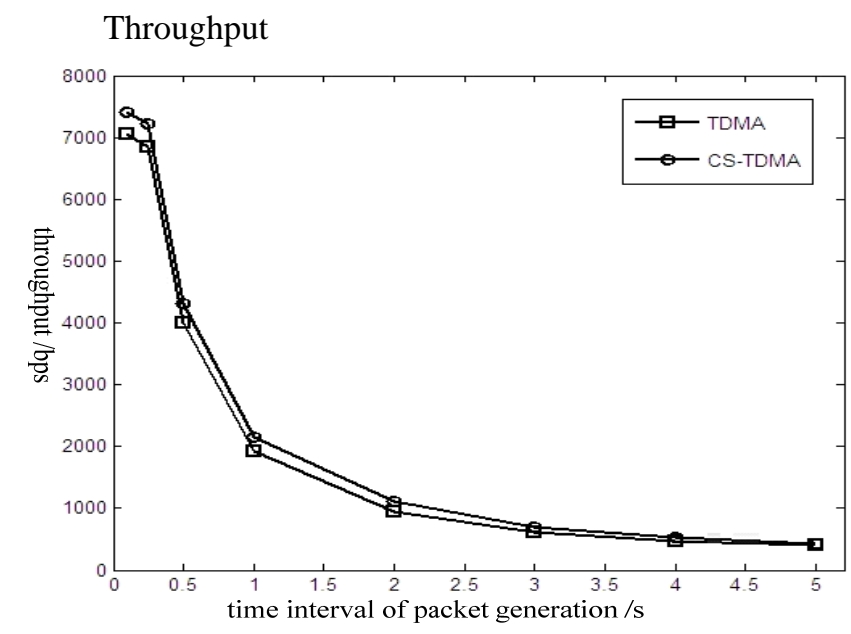

Fig.5 Comparison of network throughput

As shown in Fig.5, the network was in saturation state when the interval was under 0.25s, it was difficult to increase network throughput even if the interval time is less. And so we could get the maximal throughput was about 7200bps which was used to estimate the full capcitiy in a single slot. 
In order to ensure the protocolwas stable and effective, choosed the threshold $G_{\max }$ was 6000 bit. At the same time, when the time interval increased gradually, the throughputs of CS-TDMA and TDMA were exponential decline curves and the throughput of CS- TDMA protocol was slightly higher than TDMA protocol.

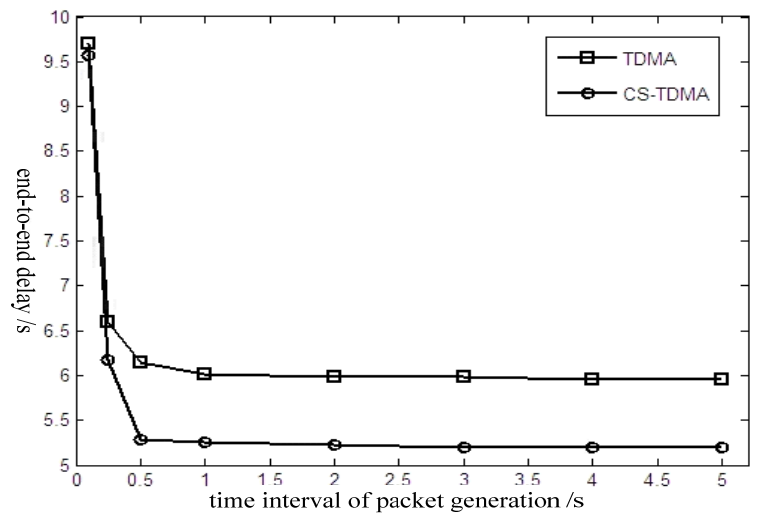

Fig.6 Comparison of end-to-end average delay

As shown in Fig.6, the average end-to-end delays were tend to be calm while the time interval was more than $0.25 \mathrm{~s}$. When the time interval was between $0.25 \mathrm{~s}$ to $0.5 \mathrm{~s}$, the delay of CS-TDMA declined fast than TDMA, because packets in CS-TDMA network also could be sent in other nodes' idle timeslot, which cutting the waiting time in queue. When time interval was greater than $0.5 \mathrm{~s}$, the delay of CS-TDMA protocol was lower than TDMA protocol about 15 percent. It showed that the CS-TDMA protocol could improve the delay performance of TDMA protocol effectively and stability.

Delay Analysis in Scenarios 2.We selected any 6 nodes as the busy-node with the interval was 0.1s. The remainders were set up different packet arrival time for $0.1 \mathrm{~s}-5 \mathrm{~s}$ in different simulation. The other conditions were same to the scenarios1.

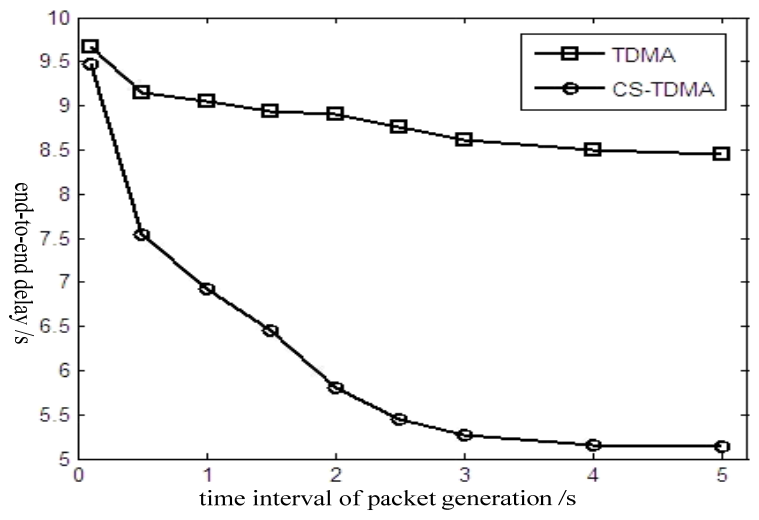

Fig.7 Comparison of end-to-end average delay

As shown in Fig.7, the average end-to-end delay of TDMA protocol was long in the condition of imbalance traffic. Although the delay decreased while the interval time of half nodes increasing, it was still high and tends to be stable at a high level. In the CS-TDMA protocol, when the business of a half nodes increased, the end-to-end delay reduced quickly and tended to be stable at a low level. So we could get the conclusion that the network used CS-TDMA protocol could utilize timeslot dynamically and had a prominent delay performance under imbalance traffic.

\section{Conclusions}

Aimed at the problem that TDMA network had a low channel using rate in an uniform traffic scene, the CS-TDMA protocol based channel sensing was proposed in this paper. Broadcasted character of wireless communication was utilized, and the goal of using time slots dynamically was achieved. The 
CS-TDMA protocol not only inherited the advantages of TDMA to ensure collision free and fair chance to send data, but also had more prominent performance in the hand of real-time than TDMA protocol. Simulation results showed that CS-TDMA protocol had better performance in message delivery ratio and throughput compared with TDMA protocol. It also had a great advantage about the network time delaycompared with TDMA protocol, especially when the traffic between the nodes was not uniform.

\section{References}

[1] SUN Jiyin, FU Guangyuan, CHE Xiaochun, in: Tactical Link Technology and System, edited by National Defense Industry Press, Beijing( 2009), in press.

[2] LIU Qinggang, in: Study on TDMA Slot Assignment based on Dynamic Priority Table, edited by Commun-ications Technology Vol.46-07(2013), p.44-49.

[3] LI Xiyang, FAN Pingzhi. Design and analysis of multi-channel MAC scheduling code for mobile ad -hoc network, edited by Journal on Communications Vol. 35-05 (2014), p.57-64.

[4] WANG Yuankun, MAO Yuquan and DING Xiaoliang: A Dynamic Slot Allocation Algorithm Based on Priority of TDMA edited by Ship Electronic Engineering Vol.29-11(2009), p. 83-86.

[5] REN Haoxiang, GUO Dawei and SHAO Ningning: A New Contention-Avoid TDMA-Based MAC Protocol edited by Chinese journal of sensors and actuators Vol.26-01(2013), p. 89-94.

[6] ADatp-22(A) Standard Operation Procedures for NATO Link22(NATO, Brussel 2006). 